\title{
Os efeitos dos impactos da economia brasileira sobre a carga tributária nacional
}

\section{The effects of the impacts of the brazilian economy on the national tax burden}

\author{
Adriana Pereira Silva Diniz ${ }^{1}$, José Washington de Freitas Diniz Filho²
}

RESUMO: A carga tributária é um agregado macroeconômico que inibe o desempenho empresarial das empresas privadas e o desenvolvimento da economia nacional e mundial. Assim, a presente pesquisa tem como objetivo principal verificar os impactos da economia brasileira sobre a carga tributária nacional. Para isso, serão utilizadas duas ferramentas estatísticas: a regressão linear múltipla e o vetor autorregressivo (VAR); serão trabalhados, como variável dependente, a carga tributária brasileira e, como variáveis independentes, o Número de Empresas Registradas na BM\&FBOVESPA, o Consumo da Administração Pública, a Formação Bruta de Capital Fixo e a Taxa de Câmbio no período de 1980 a 2015. Quanto aos resultados, observou-se que todas as variáveis independentes demonstraram promover impactos na carga tributária e estes estão munidos com testes de pressupostos confiáveis. Quanto ao comportamento da carga tributária, após os impactos dos agregados macroeconômicos, verificou-se que as variáveis Número de Empresas Registradas na BM\&FBOVESPA, Consumo da Administração Pública e Taxa de Câmbio promoveram comportamentos homogêneos na variável carga tributária; contudo, a variável Formação Bruta de Capital Fixo gerou um comportamento divergente dos demais agregados na carga tributária.

PALAVRAS-CHAVE: Carga Tributária; Economia Brasileira; Vetor Autorregressivo.

ABSTRACT: The tax burden is a macroeconomic aggregate that inhibits the corporate performance of private companies and the development of national and global economy. Thus, the main objective of this research is to verify the impacts of the Brazilian economy on the national tax burden. For this, two statistical tools will be used: multiple linear regression and autoregressive vector (VAR), where the Brazilian tax burden will be used as the dependent variable and as independent variables Number of Companies Registered at BM \& FBOVESPA, Public Administration Consumption, Gross Fixed Capital and Exchange Rate from 1980 to 2015. Regarding the results, it was observed that all the independent variables demonstrated to promote impacts on the tax burden and they are equipped with tests of reliable assumptions. Regarding the behavior of the tax burden after the impacts of the macroeconomic aggregates, it was verified that the variables Number of Companies Registered in the BM \& FBOVESPA, Public Administration Consumption and Exchange Rate promoted homogeneous behavior in the variable tax burden, however the variable Gross Fixed Capital Formation generated a divergent behavior of the other aggregates in the tax burden.

KEYWORDS: Tax Charge; Brazilian economy; Vector autoregressive.

\footnotetext{
${ }^{1}$ Acadêmica do $3^{\circ}$ período do curso de Ciências Contábeis da Universidade Ceuma e voluntária do Grupo de Pesquisa Contabilidade, Sociedade, Estado, Política e Gestão - "GPCONT” do curso de Ciências Contábeis.

E-mail: adrijwsilva@hotmail.com

2 Mestre em Administração pela Universidade Federal de Santa Maria (UFSM). Professor de Pós-Graduação e Graduação da Universidade Ceuma (Uniceuma). Líder do Grupo de Pesquisa Contabilidade, Sociedade, Estado, Política e Gestão - "GPCONT" do curso de Ciências Contábeis da Universidade Ceuma (Uniceuma). Contador da Universidade Federal do Maranhão (UFMA). E-mail: jwfdf@ hotmail.com
} 


\section{INTRODUÇÃO}

A estrutura e o comportamento do sistema tributário nacional são bastante complexos, pois envolvem elementos que vão além da elevada carga tributária até chegar aos custos causados à empresa; problema que, de longa data, apresenta intensa discussão na economia brasileira. Desde a primeira vez que se aferiu o impacto da carga tributária sobre a economia nacional, em 1947, cuja participação foi $13,8 \%$ do Produto Interno Bruto (PIB), observou-se uma clara tendência expansiva da arrecadação pública.

Conforme destaca Rosa (2007), após 1988, decorrente da promulgação do Sistema Tributário Nacional e da Constituição Federal de 1988, ocorreu um dos períodos de maior expansão da carga tributária nacional. Por outro lado, segundo Varsano (1998), movimento diferenciado, na evolução da carga tributária brasileira, verificou-se, no início da década de 1990, quando a arrecadação superou os $25 \%$ em 1991, patamar mantido até 1993, ao ser implantado, no Brasil, o novo modelo que resultaria na permissão ao país para a sustentação da estabilidade econômica.

Em linhas gerais, conforme aponta Filho (2017), esse agregado macroeconômico reflete sobre nossa economia e traz consigo algumas desvantagens, tais como: desfavorecer a produção, inibir o crescimento econômico, reduzir os investimentos; e, como consequência, lesa o poder econômico-financeiro de um país. Dessa forma, compreender o sistema tributário nacional não é tarefa fácil. O emaranhado de leis fiscais que existe, somado à burocracia inerente à cobrança do tributo, torna-se um grande ofensor para o desenvolvimento econômico do país.

Cabe apontar que a arrecadação tributária crescente reflete na economia e nas organizações como inibidores de crescimento, conforme discutem Santiago e Silva (2006), tendo na atividade agregada, nos investimentos e no consumo, sobremaneira, seus efeitos mais pronunciados. Diante do exposto, pretende-se equacionar a seguinte problemática: já que a carga tributária promove impactos na economia nacional, quais são os efeitos que a Economia Brasileira promove na Carga Tributária nacional?

Cabe destacar que o Brasil possui uma das maiores cargas tributárias do mundo. Dados do IBPT (Instituto Brasileiro de Planejamento Tributário) apontam que o país possui cerca de $33.4 \%$ da carga tributária em relação ao nosso PIB (Produto Interno Bruto), o qual é o valor de mercado de todos os bens de serviços finais produzidos dentro de uma economia pelo período de um ano.

Sachsida (2011) revela que os efeitos gerados pelo aumento da carga tributária sobre o PIB, além de ocasionar resultados negativos, implica, de forma extremamente ríspida, a economia no que se refere ao crescimento em longo prazo. Entende-se que, mais grave do que a redução do 
PIB, é o fato de que o aumento da carga tributária resulta em um impedimento para o crescimento em longo prazo da economia, no Brasil, dos estados e dos municípios.

Dessa forma, a presente pesquisa tem como objetivo principal evidenciar os impactos que a economia nacional promove na carga tributária brasileira. Para isso, será realizada uma regressão linear múltipla entre as variáveis independentes (Número de Empresas Registradas na BM\&FBOVESPA, Consumo da Administração Pública, Formação Bruta de Capital Fixo e Taxa de Câmbio) e a variável dependente (Carga Tributária Brasileira) no período de 1980 até 2015. Além disso, será utilizada a modelagem Vetor Autorregressivo (VAR) que objetiva verificar o impulsoresposta entre as variáveis macroeconômicas e a carga tributária brasileira.

Nessa esteira, Khair, Araújo e Afonso (2005) analisaram o comportamento da carga tributária e do produto agregado na economia nacional, pois confrontaram o aumento real do PIB e da receita tributária e verificaram que, no período pós-constituinte (1988-2004), o incremento da carga tributária equivaleu a 79\% do crescimento do PIB; enquanto que, no período pós-Real (19932004), a mesma relação foi de 75\%. Já no período pós-crise cambial (1998-2004), a arrecadação cresceu em valores absolutos $3 \%$ a mais do que o produto interno gerado nesse mesmo período.

Nesse ambiente, verifica-se que há inúmeros estudos realizados sobre a carga tributária brasileira, em diversas áreas e com diferentes enfoques, dada a sua importância nas decisões políticas e econômicas. Entretanto, muito se tem ainda a discutir, sobremaneira, no que tange aos impactos da arrecadação pública sobre a dinâmica da economia brasileira, em particular quando são considerados os principais agregados econômicos, aspecto esse que justifica e se constitui na principal contribuição da pesquisa.

Além disso, o presente trabalho está estruturado em quatro seções, além desta introdução. $\mathrm{Na}$ segunda, é apresentado o referencial teórico. Na terceira, os procedimentos metodológicos utilizados são mostrados. Na quarta, é relizada a análise dos resultados que foram encontrados na pesquisa e por fim, na última seção, apresentam-se as principais conclusões do trabalho.

\section{METODOLOGIA}

\subsection{Teste de Raiz Unitária}

Antes da aplicação da regressão linear múltipla e do vetor autorregressivo (VAR) sobre as variáveis macroeconômicas brasileiras, buscou-se determinar as propriedades das séries, a partir da constatação dos testes de estacionariedade, com o escopo de verificar a presença ou não de raíz 
unitária; para isso, utilizou-se o teste Augmented Dickey- Fuller (ADF). Para Bueno (2008) e Hill (2010), este teste tem, na hipótese nula, a presença de raiz unitária ou não estacionariedade da série, conforme se pode constatar na equação em (1):

$$
Y_{t}=p Y_{t-1}+u_{t}-1 \leq p \leq 1
$$

Logo, sendo $p=1$, o modelo se torna um passeio aleatório (sem deslocamento). Se $p$, de fato, for igual à unidade, se está diante do problema de raiz unitária, situação de não estacionariedade, isto é, nesse caso, a variância de $Y_{t}$ é não-estacionária.

\subsection{Regressão Linear Múltipla}

Depois de apresentada a ferramenta, que será utilizada para verificar a estacionariedade da série temporal, definiu-se o modelo analítico, o qual permite verificar quais variáveis independentes influenciam no agregado macroeconômico carga tributária, de forma a identificar os efeitos de tais relações.

A proposição sugestionada refere-se ao fato de que assim como a carga tributária nacional promove impactos na economia brasileira, conforme estudo realizado por Filho e Filho (2017), pode ser que a economia nacional promova também impactos na carga tributária brasileira.

Assim, o modelo empírico apresenta a seguinte especificação, conforme a Equação (2):

$$
Y_{i}=\beta_{0}+\beta_{1} X_{1 i}+\beta_{2} X_{2 i}+\beta_{3} X_{3 i}+\ldots+\beta_{p} X_{p i}+\varepsilon_{i}
$$

Em que $Y_{i}$ assume a variável dependente carga tributária brasileira; $X$ as variáveis independentes Número de Empresas Registradas na BM\&FBOVESPA, Consumo da Administração Pública, Formação Bruta de Capital Fixo e Taxa de Câmbio; e $\varepsilon_{t}$ corresponde ao termo de erro aleatório, o qual assume média zero e variância constante. 


\subsection{Vetor Autorregressivo (Impulso - Resposta)}

Sendo o objetivo do trabalho verificar os efeitos, na carga tributária, após os impactos da economia nacional, utilizou-se a modelagem Autoregressive Vector (VAR) que descreve a dinâmica da evolução de um conjunto de variáveis a partir de uma trajetória comum, sendo assim, consistente com um sistema de equações. Logo, nessa modelagem, todas as variáveis incluídas são consideradas endógenas. O termo autorregressivo se deve ao aparecimento do valor defasado da variável dependente no lado direito e o termo vetorial ao fato de se tratar de um vetor de duas ou mais variáveis.

Matematicamente, o modelo VAR é descrito pela equação em (3):

$$
Y_{t}=\alpha_{0}+\sum_{i=1}^{n} \alpha_{t} Y_{t-j}+\sum_{i=1}^{n} \alpha_{t} X_{t-j}+\varepsilon_{t}
$$

Em que $Y_{t}$ define a variável dependente ou exógena; $Y_{t-j}$ são os valores defasados da variável dependente; $X_{t}$ é uma matriz de variáveis incluídas no modelo: tal que $X_{t}=\left(X_{1 t}, X_{2 t}, \ldots, X_{n t}\right) ; \alpha$ é um vetor de parâmetros do modelo; e $\varepsilon_{t}$ são as perturbações aleatórias não correlacionadas entre si contemporâneas ou temporalmente, sendo que as perturbações são $\varepsilon_{t} \sim$ i.i.d. $\left(0, \sigma^{2}\right)$.

A partir da especificação do modelo VAR, torna-se possível a análise das funções impulsoresposta e da decomposição da variância, funções que permitem verificar as relações-efeitos que os agregados macroeconômicos Número de Empresas Registradas na BM\&FBOVESPA, Consumo da Administração Pública, Formação Bruta de Capital Fixo e Taxa de Câmbio exercem sobre a carga tributária.

\subsection{Decomposição da Variância}

De acordo com Enders (2010), a decomposição de variância fornece o percentual do erro da variância prevista, atribuída aos choques de uma determinada variável versus os choques nas outras variáveis do sistema. Se os choques observados, numa variável $z$, não são capazes de explicar a variância do erro de previsão da variável y, diz-se que a sequência y é exógena; caso contrário, diz-se que a sequência é endógena. 
A decomposição da variância dos erros de previsão mostra a evolução do comportamento dinâmico, apresentada pelas variáveis do sistema econômico, ao longo do tempo, isto é, permite separar a variância dos erros de previsão, para cada variável, em componentes que podem ser atribuídos por ela própria e pelas demais variáveis endógenas, isoladamente, ao apresentar, em termos percentuais, qual o efeito que um choque não antecipado, sobre determinada variável, tem sobre ela própria e as demais variáveis pertencentes ao sistema (BUENO, 2008).

\subsection{Coleta dos Dados e Construção da Base de Dados}

Para o desenvolvimento do modelo proposto, foi utilizado um conjunto de variáveis que inclui a carga tributária, o Número de Empresas Registradas na BM\&FBOVESPA, o Consumo da Administração Pública, a Formação Bruta de Capital Fixo e a Taxa de Câmbio. Todas as variáveis foram coletadas no site do Instituto de Pesquisa Econômica Aplicada, com correspondente período de 1980 a 2015, tendo periodicidade anual. Salienta-se que tal período se refere à disponibilidade integral de informações encontradas. Ademais, para a análise de efeitos reais, as séries foram deflacionadas pelo Índice Geral de Preços - Disponibilidades Internas - e calculadas pela Fundação Getúlio Vargas. As séries, como também as definições e as unidades de medida, estão expostas no Quadro 1.

Quadro 1: Variáveis, definições e unidades de medidas

\begin{tabular}{|ccc|}
\hline VARIÁVEL & UNIDADE DE MEDIDA & $\begin{array}{c}\text { DEFINIÇOES UTILIZADAS } \\
\text { NO MODELO EMPÍRICO }\end{array}$ \\
\hline Carga tributária & $\%$ & CT \\
Número de empresas registradas na & Unidades & BM\&FBOVESPA \\
BM\&FBOVESPA & R \$ milhões ou bilhões & CAP \\
Consumo da administração pública & $\mathrm{R} \$$ milhões ou bilhões & FBCF \\
Investimento & $\%$ & TC \\
\hline
\end{tabular}

Fonte: Elaborada pelos autores.

\section{RESULTADOS E ANÁLISE DOS RESULTADOS}

\subsection{Análise dos Efeitos na Carga Tributária Após Impactos da Economia Brasileira}

Para atender aos objetivos propostos, seguiu-se um conjunto de procedimentos estatísticos. A partir disso, inicialmente, averiguou-se a ordem de integração das séries utilizadas por meio dos testes de estacionariedade. A estacionariedade ou não das séries macroeconômicas foi verificada a 
partir da utilização do teste Dickey-Fuller Aumentado (ADF) em nível e em diferença; sendo que os resultados estão expostos na Tabela 1.

De forma geral, os resultados indicam que todas as variáveis: Carga Tributária, Número de Empresas Registradas na BM\&FBOVESPA, Consumo da Administração Pública, Formação Bruta de Capital Fixo e Taxa de Câmbio são não estacionárias em nível. Logo, possuem raiz unitária, ao considerar-se o nível de 5\% de significância. Nesse sentido, fez-se necessário estimar as séries em primeira diferença, procedimento que gerou resultados indicativos de que inexiste raiz unitária; dessa forma, as séries são estacionárias ao nível de 5\% de significância, portanto consideradas integradas de ordem um I(1).

Tabela 1: Resultados do teste de raiz unitária (ADF) para as séries econômicas analisadas entre 1980 e 2015 - em nível e em diferença.

\begin{tabular}{lcllccl}
\hline \multirow{2}{*}{ Variável } & \multicolumn{2}{c}{ Em nível } & \multicolumn{3}{c}{ Em diferença } \\
\cline { 2 - 7 } & Def. & $\begin{array}{l}\text { Estatística } \\
\text { Teste }\end{array}$ & $\begin{array}{l}\text { Valor } \\
\text { Crítico (5\%) }\end{array}$ & Def. & $\begin{array}{l}\text { Estatística } \\
\text { Teste }\end{array}$ & $\begin{array}{l}\text { Valor } \\
\text { Crítico (5\%) }\end{array}$ \\
\hline CT & 8 & $-2,5355^{*}$ & -3.5577 & 8 & $-5,6688^{*}$ & $-4,2967$ \\
BM\&FBOVESPA & 8 & $-2,4796^{*}$ & -3.5577 & 8 & $-5,2024^{*}$ & $-4,2967$ \\
CAP & 8 & $-2,6421^{*}$ & -3.5577 & 8 & $-5,4163^{*}$ & $-4,2967$ \\
FBCF & 8 & $-2,4796^{*}$ & -3.5577 & 8 & $-5,2024^{*}$ & $-4,2967$ \\
TC & 8 & $-2,8971^{*}$ & -3.5577 & 8 & $-3,6939^{*}$ & $-4,2845$ \\
\hline
\end{tabular}

Fonte: Resultados da pesquisa com base no software Gretl. Elaborada pelos autores.

Após verificar-se o comportamento da estacionariedade das variáveis macroeconômicas utilizadas na pesquisa, agora o trabalho visa identificar quais variáveis influenciam no comportamento da carga tributária brasileira.

O primeiro passo, em nosso estudo, é verificar o teste $\mathrm{F}$ de significância global, pois este objetiva perceber se a modelagem que se utiliza é útil para explicar a influência das variáveis Número de Empresas Registradas na BM\&FBOVESPA, Consumo da Administração Pública, Formação Bruta de Capital Fixo e Taxa de Câmbio sobre a variável dependente Carga Tributária.

Conforme se observa na tabela 02 , o valor $\mathrm{P}$ do teste $\mathrm{F}$ do modelo apresenta um valor de 0,001819; isso significa, estatisticamente, que pelo menos uma das variáveis macroeconômicas impacta na carga tributária nacional e que a modelagem pode ser utilizada na pesquisa.

Logo em seguida, verificou-se o teste de significância individual com o objetivo de perceber o grau de influência de cada variável independente sobre a carga tributária. Sendo assim, para que uma variável independente explique a variável dependente, o seu $\mathrm{P}$ valor tem que ser menor que $5 \%$.

Assim, na tabela 02, constata-se que as variáveis independentes Número de Empresas Registradas na BM\&FBOVESPA, Consumo da Administração Pública, Formação Bruta de Capital 
Fixo e Taxa de Câmbio são estatisticamente significantes, já que o Valor P é menor que o nível de significância de 0,05. Dessa forma, rejeita-se a hipótese nula e conclui-se que estas variáveis são estatisticamente significativas e que influenciam o comportamento da carga tributária nacional, pois apresentam um P valor de 0,0325, 0,0351, 0,0032 e 0,0423, respectivamente.

Cabe apontar que a variável Consumo da Administração Pública apresenta um sinal $\beta$ positivo, o que significa que há uma relação direta entre essa variável macroeconômica e a carga tributária.

Entretanto, comportamento contrário foi observado nas variáveis Número de Empresas Registradas na BM\&FBOVESPA, Formação Bruta de Capital Fixo e Taxa de Câmbio, já que estas apresentaram um coeficiente negativo, pois informou-se que há uma relação inversa entre elas e a carga tributária.

O próximo passo foi verificar os valores de $\mathrm{R}^{2}$ e $\mathrm{R}^{2}$ ajustado, em que o escopo foi identificar quanto as variáveis Número de Empresas Registradas na BM\&FBOVESPA, Consumo da Administração Pública, Formação Bruta de Capital Fixo e Taxa de Câmbio explicam a carga tributária. Conforme resultados encontrados, na tabela 02 , as variáveis independentes explicam $43 \%$ da variável carga tributária.

Tabela 02: Resultados do Modelo de Regressão com erros-padrão Robustos

\begin{tabular}{lcccll}
\hline \multicolumn{1}{c}{ Variáveis } & $\boldsymbol{\beta}$ & $\mathbf{t}$ & Valor $\mathbf{P}$ & Número de Observações & 35 \\
\cline { 1 - 4 } BM\&FBOVESPA & $-0,0297295$ & $-2,2539$ & 0,0325 & $\mathrm{~F}(4,27)$ & 5,716702 \\
CAP & $1,2566 \mathrm{e}-05$ & 2,2190 & 0,0351 & $\mathrm{P}-$ valor $(\mathrm{F})$ & 0,001819 \\
FBCF & $-6,79759 \mathrm{e}-06$ & $-3,2371$ & 0,0032 & $\mathrm{R}^{2}$ & 0,427751 \\
TC & $-0,327544$ & $-2,1310$ & 0,0423 & $\mathrm{R}^{2}$ Ajustado & 0,342974 \\
Constante & $-0,0771564$ & $-0,2459$ & 0,8076 & \\
\hline \multicolumn{7}{l}{ Fonte: Resultados da pesquisa com base no software Gretl. Elaborada pelos autores. }
\end{tabular}

Fonte: Resultados da pesquisa com base no software Gretl. Elaborada pelos autores.

Por fim, foi empregado, ao modelo estatístico, o teste de Heteroscedasticidade de White, tendo por objetivo verificar a constância da Variância dos resíduos. Além disso, complementarmente, foram aplicados à modelagem os testes de Normalidade dos resíduos e de Multicolinearidade para constatar se as variáveis não fornecem informações semelhantes.

Ao observar-se a tabela 03, descobriu-se que o modelo não apresenta problemas de Heteroscedasticidade, pois o seu Valor P registra uma importância de 0,10 e o nível de significância é acima de 0,05. Isso significa que a variância, entre os resíduos, mantém-se em todo o espectro das variáveis independentes e há Homocedasticidade na variância dos resíduos. 
No teste de Normalidade, achou-se um Valor P de 0,28, o que significa que, na modelagem apresentada, não se rejeita a hipótese nula, pois se trata de uma distribuição normal e, assim, se cumpre o pressuposto de normalidade, já que o nível de significância é de até 0,05.

Quanto ao teste de Multicolinearidade, encontrou-se uma média entre as três variáveis independentes de 2,35 do VIF (fatores de inflação da variância), o que leva a concluir que os resultados da regressão não apresentam problemas de Multicolinearidade. Conforme Gujarati (2006), quanto maior o valor do VIF de uma variável independente, maior a Colinearidade dessa variável.

Tabela 03: Testes dos Pressupostos

\begin{tabular}{c|c|c|c}
\hline Pressupostos & Ho & Valor P & $\begin{array}{c}\text { Nível de } \\
\text { Significância }\end{array}$ \\
\hline Normalidade dos resíduos & Normalidade dos resíduos & 0,29 & 0,05 \\
\hline Heteroscedasticidade de White & Variância dos resíduos é constante & 0,10 & 0,05 \\
\hline Multicolinearidade & $\begin{array}{c}\text { FIV < 10 não há problema de } \\
\text { Multicolinearidade }\end{array}$ & 2,34 & N/A \\
\hline
\end{tabular}

Fonte: Resultados da pesquisa com base no software Gretl. Elaborada pelos autores.

Depois de analisadas as propriedades estatísticas das séries, procedeu-se a estimação da modelagem VAR, a partir da qual se obtiveram as funções de resposta a impulsos e à decomposição da variância dos erros de previsão. Os resultados das referidas funções, conforme Figura 1, foram obtidos, pois se consideraram as séries em diferença e a utilização do choque de um desvio padrão.

Figura 1: Resultados das funções impulso - resposta da Carga tributária a um choque nas Variáveis Macroeconômicas, Número de Empresas Registradas na BM\&FBOVESPA, Consumo da Administração Pública, Formação Bruta de Capital Fixo e Taxa de Câmbio.

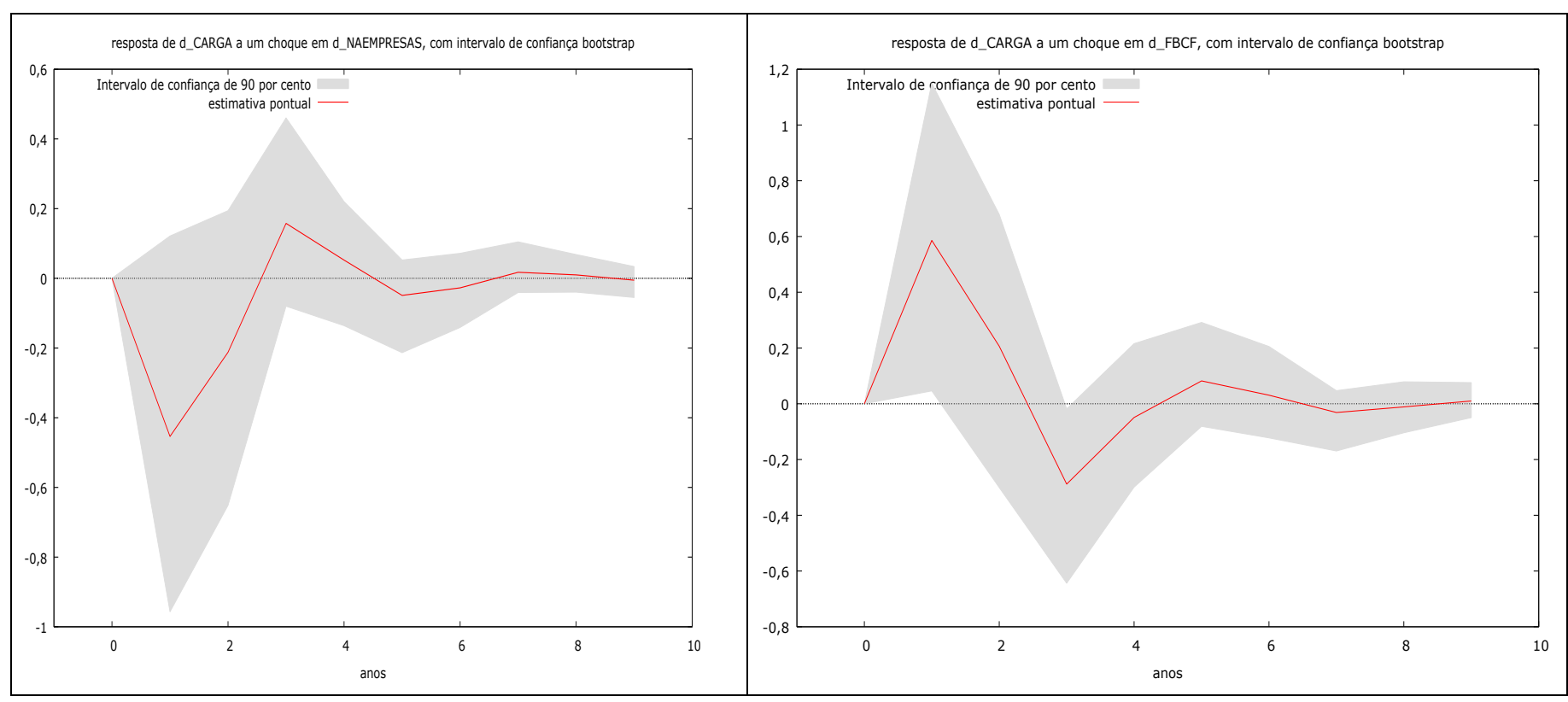




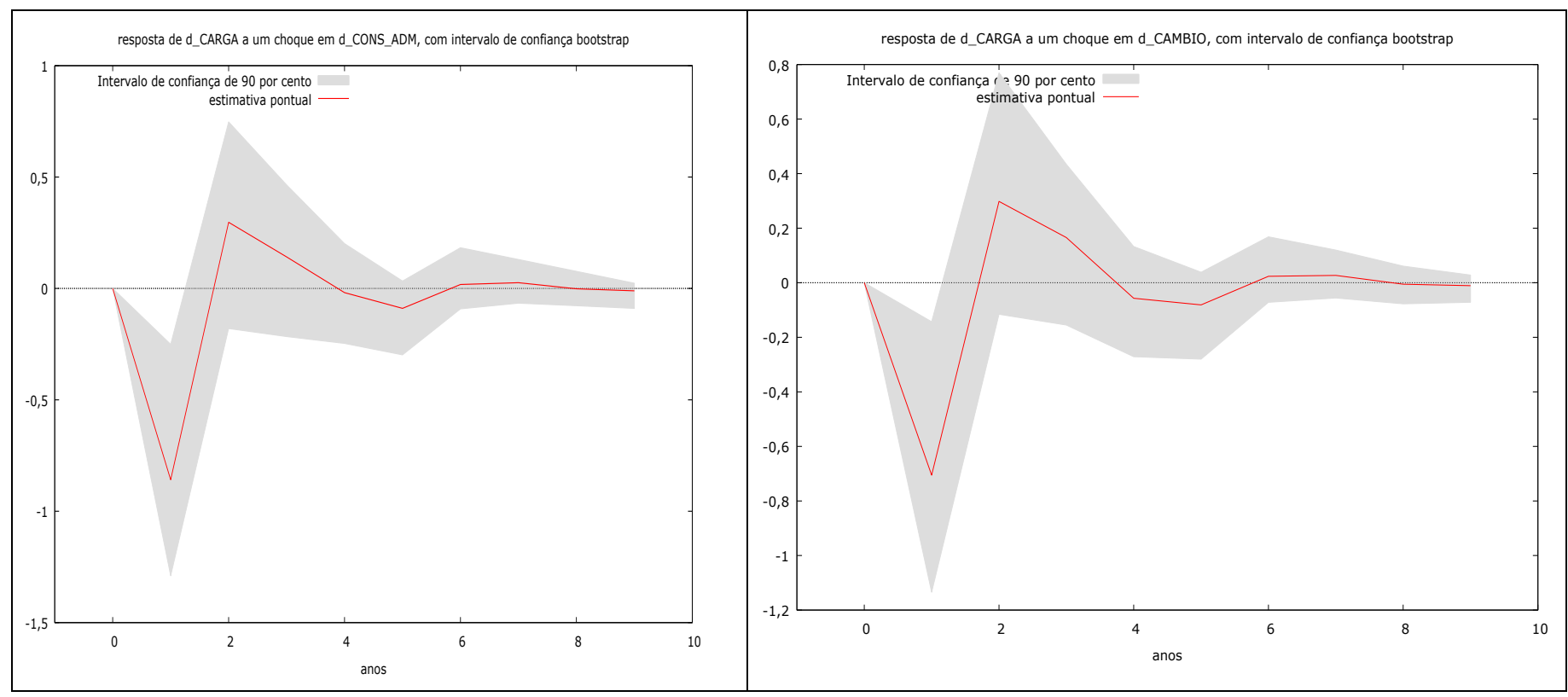

Fonte: Resultados da pesquisa com base no software Gretl. Elaborada pelos autores.

Ao fazer-se uma análise geral da reação da Carga Tributária, após os impactos nos agregados macroeconômicos, constata-se que a maioria das variáveis, no caso o Número de Empresas Registradas na BM\&FBOVESPA, Consumo da Administração Pública e Taxa de Câmbio, provocou um comportamento decrescente acentuado na variável carga tributária no primeiro período. Entretanto, a variável Formação Bruta de Capital Fixo fez com que a carga tributária apresentasse, no primeiro ano, um comportamento ascendente.

Ao partir-se para uma análise individualizada, após os impactos nos agregados macroeconômicos sobre a carga tributária, inicia-se com a variável Número de Empresas Registradas na BM\&FBOVESPA, a qual promoveu na carga tributária, conforme figura 01, uma tendência decrescente nos períodos de 0 a 1 e de 3 a 5. Porém, no período de 1 a 3, constata-se um comportamento ascendente da carga tributária e um período de constância a partir do quinto período.

Quanto ao comportamento da carga tributária, após os impactos na variável Formação Bruta de Capital Fixo, constata-se uma tendência adversativa em relação às demais variáveis macroeconômicas. Nos períodos de 0 a 1 e 3 a 5, a carga tributária demonstrou um comportamento ascendente; contudo, no período de 1 a 3 , foi observado um comportamento abrupto decrescente. A partir do período 5, percebeu-se uma constância na tendência da variável carga tributária, conforme tabela 01 .

Nessa linha, Francini (2013) sugere que o pleno desenvolvimento de uma economia necessita de uma mudança na realocação dos recursos disponíveis em direção à ampliação dos investimentos. Esta ampliação e a consequente sustentação de taxas elevadas de crescimento 
exigem alterações na dinâmica dos gastos correntes do Governo, com o objetivo de ampliar o investimento público, limitar a expansão da carga tributária e manter a taxa de juros em níveis internacionais.

Na variável Consumo da Administração Pública, o comportamento da carga tributária não foi muito diferente da variável anterior, pois apresentou um comportamento decrescente nos período de 0 a 1 e de 2 a 5. Contudo, no período de 1 a 2, constatou-se um comportamento ascendente da carga tributária, conforme tabela 01, e uma tendência constante a partir do quinto período.

Sobre essa discussão, Pereira (2010), ao analisar a incidência da atividade do setor público, com ênfase para a política fiscal, sobre a distribuição de renda, evidencia que tão importante quanto os efeitos dos tributos são, também, os efeitos dos gastos do Governo. Nesse sentido, para o apropriado funcionamento do Estado, é essencial a gestão adequada das finanças públicas.

Para Sachsida (2011), do ponto de vista econômico, o crescimento estatal gera a necessidade do aumento da carga tributária. Contudo, o aumento desta torna o recebimento dos bens públicos mais onerosos para os trabalhadores; consequentemente, há a necessidade de despender mais horas de trabalho para pagar o maior nível de impostos.

Para a última variável impactada, no caso a Taxa de Câmbio, ao verificar-se o que ocorre com o agregado macroeconômico carga tributária, foi observado o mesmo comportamento que nas variáveis Número de Empresas Registradas na BM\&FBOVESPA e Consumo da Administração Pública, ou seja, um comportamento decrescente nos períodos de 0 a 1 e de 2 a 5 . Entretanto, constatou-se uma tendência ascendente no período de 1 a 2 e uma constância a partir do período 5 .

Ao explicar o exposto, Filho (2017) destaca que a depreciação cambial, resultante de choques na tributação, pode denotar as dificuldades da economia em conter os desequilíbrios do setor público. Em outras palavras, refletiria a desconfiança externa em relação à solidez da economia. Entretanto, a análise isolada dessa relação é limitada, dado que o entendimento passaria por um exame conjunto de diversos aspectos, os quais envolveriam, além da política fiscal, as políticas monetária e cambial. Todavia, o que se pode discutir é que políticas fiscais expansionistas de elevação de alíquotas, ao impor custos adicionais, diminuiriam a produção e, por sua vez, os fluxos de comércio seriam afetados pela redução da competitividade dos produtos domésticos.

Por fim, em continuidade à análise, tem-se a decomposição da variância dos erros de previsão (ver Figura 02), a qual fornece a participação do erro da variância (prevista), atribuída aos choques de uma determinada variável sobre as outras variáveis do sistema. Quanto à carga tributária, observa-se que, nos primeiros períodos, a variância é explicada, em grande medida, pelo 
próprio comportamento da tributação. Ao longo do tempo, ganham importância o consumo da administração pública, a taxa de câmbio e a formação bruta de capital fixo. Já a variável número de empresas registradas na BM\&FBOVESPA apresentou efeito reduzido sobre os erros de previsão da carga tributária brasileira.

Figura2: Resultados da Decomposição da Variância dos Erros de Previsão da Carga Tributária

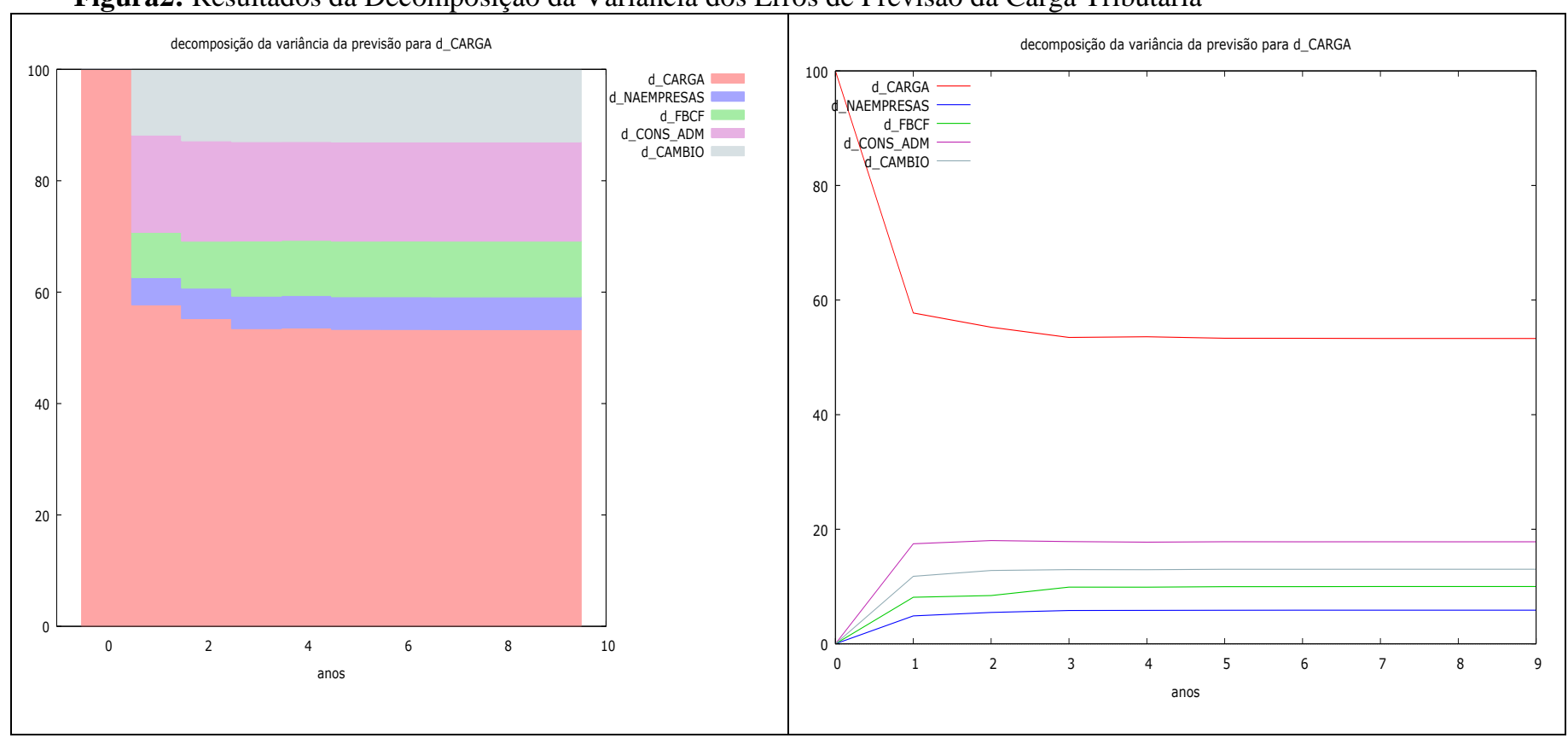

Fonte: Resultados da pesquisa com base no software Gretl. Elaborada pelos autores.

\section{CONSIDERAÇÕES FINAIS}

A estrutura e o comportamento do sistema tributário nacional são bastante complexos, sendo que envolvem elementos que vão além da elevada carga tributária até chegar aos custos causados à empresa; problema que, de longa data, apresenta intensa discussão na economia brasileira.

Diante do exposto, a presente pesquisa tem com objetivo principal evidenciar os impactos que a economia nacional promove na carga tributária brasileira. Para isso, realizou-se uma regressão linear múltipla entre as variáveis independentes (Número de Empresas Registradas na BM\&FBOVESPA, Consumo da Administração Pública, Formação Bruta de Capital Fixo e Taxa de Câmbio) e a variável dependente (Carga Tributária Brasileira) no período de 1980 até 2015. Além disso, utilizou-se a modelagem Vetor Autorregressivo (VAR) que objetivou verificar o impulsoresposta entre as variáveis macroeconômicas utilizadas na pesquisa e a carga tributária brasileira. 
Quanto aos resultados da regressão múltipla, constatou-se que as variáveis independentes Número de Empresas Registradas na BM\&FBOVESPA, Consumo da Administração Pública, Formação Bruta de Capital Fixo e Taxa de Câmbio demonstraram, num primeiro momento, impactar na carga tributária brasileira, uma vez que todos os $\mathrm{p}$ valores apresentaram valores abaixo de 0,05 .

Além disso, com o fito de corroborar os impactos da economia brasileira sobre a carga tributária nacional e verificar os efeitos desses impactos, aplicou-se, sobre as variáveis estudadas, o VAR, a partir do qual se obtiveram as funções de resposta a impulsos. Nos resultados, observou-se que as variáveis Número de Empresas Registradas na BM\&FBOVESPA, Consumo da Administração Pública e Taxa de Câmbio fizeram com que a carga tributária brasileira apresentasse, nos primeiros períodos, uma tendência decrescente; depois iniciava-se uma comportamento ascendente e, por fim, uma constância em seu desenvolvimento. Comportamento contrário foi verificado no impacto da variável Formação Bruta de Capital Fixo sobre a carga tributária, já que, nos primeiros períodos, houve uma tendência crescente na variável carga tributária, mas depois um abrupto declínio no comportamento desta até apresentar uma tendência de uniformidade no comportamento da variável.

Sendo assim, estudos, cada vez mais aprofundados sobre os efeitos e o comportamento do agregado macroeconômico carga tributária, podem gerar ferramentas de controle e administração desse grande inibidor de crescimento da economia brasileira e mundial para os gestores públicos e privados. Para a realização de novas pesquisas, sugere-se a utilização de investigações mais específicas nos efeitos desse agregado na área empresarial e também na área pública.

\section{REFERÊNCIAS}

ALEXANDRINO, M.; PAULO, V. Manual de direito tributário. Rio de Janeiro: Impetus, 2006.

BUENO, R. L. S. Econometria de séries temporais. São Paulo: Cengage Learning, 2008.

ENDERS, W..Applied econometric time series. New York: John Wiley and Sons, 2010.

FILHO, J.W.; FILHO, R. B. UMA ANÁLISE DO COMPORTAMENTO E DOS EFEITOS DA CARGA TRIBUTÁRIA NA ECONOMIA BRASILEIRA NO PERÍODO DE 1980 A 2012. RAGC, v.5, n.20, p.45-64. Minas Gerais: 2017.

FILHO, J. W. F. D.. A Carga Tributária Na Economia Brasileira. Editora Lexia: São Paulo, 2017. 
FRANCINI, P. A questão tributária como obstáculo ao desenvolvimento.Interesse Nacional, ano 5, n. 20, jan./mar. 2013. Disponível em: <http://interessenacional.uol.com.br/index.php/edicoesrevista/a-questao-tributaria-como-obstaculo-ao-desenvolvimento/>. Acesso em: 28 fev. 2014

HARADA, K. Direito financeiro e tributário. 20. ed. São Paulo: Atlas, 2012.

HILL, R. C.. Econometria. São Paulo: Saraiva, 2010

KHAIR, A.; ARAUJO, E. A.; AFONSO, J. R. R.. Tributos e juros: as duas grandes cargas na economia. 2005. Disponível em:

<www.frpii.org/english/Portals/0/.../tributos_y_juros_afonso_feb_05.pdf. Acesso em: 14 de março de 2014

LOPES, M. L. R. Direito tributário brasileiro. Rio de Janeiro: Impetus, 2009.

PEREIRA, J. M. Gestão das políticas fiscal e monetária: os efeitos colaterais da crise mundial no crescimento da economia brasileira. Observatório de la Economia Latinoamericana, n. 148, 2010. Disponível em: http://www.eumed.net/cursecon/ecolat/br/11/jmp.htm>. Acesso em: 17 mar. 2018.

ROSA, D. D. A evolução da carga tributária no Brasil. Âmbito Jurídico, 2007. Disponível em: <www. ufsm. br/direito/artigos/tributário/ ..., 2006 - egov.ufsc.br>. Acesso em: 15 de novembro de 2017.

SASCHIDA, A. Como os Impostos Afetam o Crescimento Econômico?. Brasil: Economia e Governo, 2011. Disponível em: <http://www.brasil-economiagoverno. org.br/2011/03/16/como-osimpostos-afetam-o-crescimento-economico/ > . Acesso em: $01 \mathrm{dez} .2017$.

SANTIAGO, M. F.; SILVA, J. L. G. Evolução e composição da carga tributária brasileira. Revista Brasileira de Gestão e Desenvolvimento Regional, Taubaté, v. 2, n. 1, p. 22-41, jan./abr. 2006.

SABBAG, E. Manual de direito tributário. 4. ed. São Paulo: Saraiva, 2012.

SANTIAGO, M. F.; SILVA, J. L. G. Evolução e composição da carga tributária brasileira. Revista Brasileira de Gestão e Desenvolvimento Regional, Taubaté, v. 2, n. 1, p. 22-41, jan./abr. 2006.

SASCHIDA, A. Como os Impostos Afetam o Crescimento Econômico? Brasil: Economia e Governo, 2011. Disponível em: <http://www.brasil-economia-governo.org.br/2011/03/16/como-osimpostos-afetam-o-crescimento-economico/ > . Acesso em: 01 dez. 2013.

VARSANO, R. et al. Uma análise da carga tributária do Brasil. Texto para discussão, Rio de Janeiro, n. 583, 1998. 